\title{
Phase and Frequency Matching-based Signal Processing Method for Coriolis Mass Flowmeters
}

\author{
Yaqing Tu, Yanlin Shen, Haitao Zhang, Ming Li \\ Department of Information Engineering, Logistical Engineering University, Chongqing 401311, People's Republic of China, \\ yq.tu@163.com
}

\begin{abstract}
Signal processing precision of Coriolis mass flowmeters affects the measurement accuracy directly. To improve the measurement accuracy of Coriolis mass flowmeters, a phase and frequency matching-based signal processing method for Coriolis mass flowmeters is proposed. Estimated phase difference is obtained by means of frequency estimation, $90^{\circ}$ phase shift, generating reference signals and crosscorrelation. Simulated results demonstrate that the proposed method has better phase difference estimation and anti-interference performance than the Hilbert transform method, cross-correlation method, data extension-based correlation method, and quadrature delay estimator. Measurement results of Coriolis mass flowmeters verify the effectiveness and superiority of the proposed method in practice.
\end{abstract}

Keywords: Coriolis mass flowmeters, phase and frequency matching, phase difference, frequency estimation, $90^{\circ}$ phase shift.

\section{INTRODUCTION}

Coriolis mass flowmeters (CMF) have attracted considerable attention because they are widely applied in numerous industries such as petroleum, chemistry, and pharmacy. CMF are a kind of high-precision flowmeters which can measure the mass flow rates of the fluid directly. CMF obtain mass flow rates by means of measuring phase difference or time delay between two vibration signals detected by electromagnetic sensors [1]-[3]. Therefore, accurate phase difference estimation is significantly important and necessary for CMF.

To obtain estimated phase difference, the discrete Fourier transform (DFT) method [4]-[5] gets frequency estimation by calculating DFT of two signals and gains phase difference estimation by the subtraction of two DFT phases at the estimated frequency. The DFT method has good noise immunity. But the inherent defect of DFT called spectrum leakage brings the estimation error of phase difference, especially for short sinusoidal signals. The cross-correlation (CC) method [6]-[8] gets estimated phase difference by means of cross-correlation and auto-correlation of two signals. This method is computationally efficient and can obtain phase difference without the information of signal frequency. However, its anti-interference performance is poor and its phase difference estimation is biased, which is caused by non-integral period sampling. To suppress the effect of non-integral period sampling on phase difference estimation, the data extension-based correlation (DEC) method [9] extends the signals to approach integral period sampled signals by two signals' truncation or shift and obtains estimated phase difference by employing the crosscorrelation method. Thus, this method improves the estimation performance of phase difference when sampled signals are of non-integral period, whereas its antiinterference performance is still poor because of the noise variance of auto-correlation signals. To improve the antiinterference performance of phase difference estimation, the dual quadrature demodulation (DQD) method [2] obtains phase difference estimation by means of cross-correlation between sampled signals and two generated signals, lowpass filter. This method is independent of the amplitudes of sampled signals and has suppressed the effect of frequency doubling terms on phase difference estimation in some extent. However, accurate frequency estimation is needed to ensure good estimation precision. The Hilbert transform (HT) method [10]-[12] completes $90^{\circ}$ phase shift of sinusoidal signals by their Hilbert transform. Then, estimated phase difference is calculated by cross-correlation of sinusoidal signals and their Hilbert transform. Compared with the CC method and DEC method, the HT method has better anti-interference performance. However, end effects of Hilbert transform resulting from non-integral period sampled signals lead to the bias of the HT method. The quadrature delay estimator [13]-[15] (QDE) obtains quadrature-phase components of sampled signals by their time-shifting and gets phase difference by means of cross- 
correlation of in-phase and quadrature-phase components. The QDE method has good estimation performance when $90^{\circ}$ phase shift is exactly performed by choosing the sampling period properly, whereas the QDE method shows bias if the introduced phase shift is not exactly $90^{\circ}$.

To improve the measurement accuracy of $\mathrm{CMF}$, a phase and frequency matching-based signal processing method for Coriolis mass flowmeters is proposed, which is expected to eliminate the dependence of phase difference estimation on integral period sampling and improve its estimation precision. The rest of this paper is organized as follows: in section 2, the key idea of phase and frequency matchingbased signal processing method for Coriolis mass flowmeters is developed. The proposed method is validated by simulations and experiments in section 3 and 4 by comparing with the CC, HT, DEC and QDE methods. Finally, conclusions are drawn in section 5.

\section{METHOD DEVELOPMENT}

To improve the signal processing precision of $\mathrm{CMF}$, a phase and frequency matching-based signal processing method for Coriolis mass flowmeters is proposed. The main steps of the proposed method are shown in Fig.1. Firstly, the CMF signals are sampled and two signals are obtained. Secondly, frequency estimation of two CMF signals is obtained by means of auto-correlation, generating reference signals, constructing error function and calculating its minimum. Thirdly, phase difference estimation is obtained by means of $90^{\circ}$ phase shift, generating reference signals and cross-correlation. Finally, the mass flow rate is calculated according to estimated phase difference.

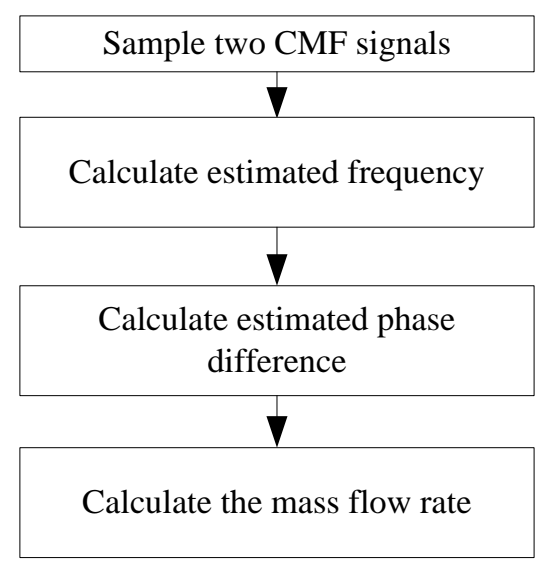

Fig.1. The main step of the proposed method.

Two noisy real sinusoidal signals for $\mathrm{CMF}$ are as follows:

$$
\left\{\begin{array}{l}
x(t)=s_{x}(t)+z_{x}(t) \\
y(t)=s_{y}(t)+z_{y}(t)
\end{array}\right.
$$

where $s_{x}(t)=A \cos \left(\omega t+\theta_{1}\right)$ and $s_{y}(t)=B \cos \left(\omega t+\theta_{2}\right)$ are noise-free signals, $\omega$ is the sinusoidal frequency, $A$ and $B$, $\theta_{1}$ and $\theta_{2}$ are sinusoidal amplitudes and initial phases, respectively, $z_{x}(t)$ and $z_{y}(t)$ are additive white Gaussian noise.

The CMF signals are sampled and two signals are obtained as follows:

$$
\left\{\begin{array}{l}
x=\{x(1), x(2) \cdots x(N)\} \\
y=\{y(1), y(2) \cdots y(N)\}
\end{array} \quad n=1,2 \cdots N\right.
$$

where $N$ is the sinusoidal length, $x(n)=s_{x}(n)+z_{x}(n)$, $y(n)=s_{y}(n)+z_{y}(n)$.

For sinusoidal signals $s(n)=A \cos (n \omega+\theta)$, we can get the following equation according to linear prediction property of sinusoidal signals:

$$
s(n-b)-s(n+b)=2 \sin (b \omega) \hat{s}(n)
$$

Based on (3), the $n$th quadrature-phase component of sampled signals $s(n)$ is calculated as follows:

$$
\hat{s}(n)=\frac{s(n-b)-s(n+b)}{2 \sin (b \omega)}
$$

where $b=\operatorname{round}(0.5 \pi / \hat{\omega}), \operatorname{round}(t)$ represents the integer which is the closest to $t$.

According to (4), the quadrature-phase components of $x(n)$ and $y(n)$ are calculated as follows:

$$
\left\{\begin{array}{l}
\hat{x}(n)=\frac{x(n-b)-x(n+b)}{2 \sin (b \omega)} \\
\hat{y}(n)=\frac{y(n-b)-y(n+b)}{2 \sin (b \omega)}
\end{array}\right.
$$

Equation (5) indicates that signal frequency $\omega$ should be known to obtain $\hat{x}(n)$ and $\hat{y}(n)$. Therefore, to obtain signal frequency $\omega$, the $k$ th auto-correlation $\operatorname{signal} r(k)$ is calculated,

$$
r(k)=\sum_{n=p+1}^{N-p}\left\{\begin{array}{l}
{[x(n+k)+x(n-k)] x(n)} \\
+[y(n+k)+y(n-k)] y(n)
\end{array}\right\}
$$

where $k$ is the lag of auto-correlation signals, $k=1,2, \cdots p$, $p=\operatorname{round}(0.46 N)$.

The expectation value of $r(k)$ is

$$
E[r(k)]=\cos (k \omega) \sum_{n=p+1}^{N-p} 2\left[s_{x}^{2}(n)+s_{y}^{2}(n)\right]
$$


Equation (7) indicates that auto-correlation signals $\mathbf{r}$ have the characteristics that $\mathbf{r}$ have zero initial phase and the same frequency with two CMF signals.

Based on the characteristics of $\mathbf{r}$, reference signals $\lambda$ are generated.

$$
\lambda=\{\cos (\hat{\omega}), \cos (2 \hat{\omega}), \cos (3 \hat{\omega}) \cdots \cos (K \hat{\omega})\}
$$

The error function between auto-correlation signals $\mathbf{r}$ and reference signals $\lambda$ is constructed as follows:

$$
J(\hat{\omega})=\sum_{k=1}^{K} r^{2}(k) \sum_{k=1}^{K} \lambda^{2}(k)-\left(\sum_{k=1}^{K} r(k) \lambda(k)\right)^{2}
$$

According to Cauchy inequation, we have $J(\hat{\omega}) \geq 0$. When $\mathbf{r}=q \boldsymbol{\lambda}$ ( $q$ is a constant), that is to say, autocorrelation signals $\mathbf{r}$ and reference signals $\lambda$ have the same initial phase and frequency, the error function $J(\hat{\omega})$ attains its minimum. To obtain $\hat{\omega}, \lambda(k)=\cos (k \hat{\omega})$ is expanded into Taylor series at coarse estimation frequency $\omega_{1}$, whose first order is preserved and high level terms are ignored.

$$
\lambda(k)=\cos (k \hat{\omega})=\cos \left(k \omega_{1}\right)-k \sin \left(k \omega_{1}\right)\left(\hat{\omega}-\omega_{1}\right)
$$

Substituting $\lambda(k)$ into (9) and making the derivation of $J(\hat{\omega})$ equal zero yields

$$
\hat{\omega}=\omega_{1}+\frac{\left\{\left(\begin{array}{l}
\left(\sum_{k=1}^{K} r(k) \cos \left(k \omega_{1}\right) \sum_{k=1}^{K} r(k) k \sin \left(k \omega_{1}\right)\right) \\
-\sum_{k=1}^{K} r^{2}(k) \sum_{k=1}^{K}\left[k \cos \left(k \omega_{1}\right) \sin \left(k \omega_{1}\right)\right]
\end{array}\right\}\right.}{\left(\sum_{k=1}^{K} r(k) k \sin \left(k \omega_{1}\right)\right)^{2}-\sum_{k=1}^{K} r^{2}(k) \sum_{k=1}^{K}\left[k \sin \left(k \omega_{1}\right)\right]^{2}}
$$

Equation (5) shows that when $\hat{\omega}=\omega$, the expectation of $\hat{x}(n)$ and $\hat{y}(n)$ can be expressed as follows:

$$
\left\{\begin{array}{l}
E[\hat{x}(n)]=A \sin \left(n \omega+\theta_{1}\right) \\
E[\hat{y}(n)]=B \sin \left(n \omega+\theta_{2}\right)
\end{array}\right.
$$

where $1+b \leq n \leq N-b$.

To obtain phase difference estimation of two CMF signals, two reference signals $h(n)$ and $\hat{h}(n)$ are generated as follows:

$$
\left\{\begin{array}{l}
h(n)=\cos (n \hat{\omega}) \\
\hat{h}(n)=\sin (n \hat{\omega})
\end{array}\right.
$$

$h(n)$ and $\hat{h}(n)$ have the same frequency of two CMF signals and their quadrature-phase components. Besides, the initial phase of $h(n)$ is zero and the phase difference of $h(n)$ and $\hat{h}(n)$ is $\pi / 2$.

Two cross-correlation signals are calculated through the cross-correlation of two CMF signals $x(n)$ and $y(n)$, their quadrature-phase components $\hat{x}(n)$ and $\hat{y}(n)$, two reference signals $h(n)$ and $\hat{h}(n)$.

$$
\left\{\begin{array}{l}
R_{1}=a_{11} a_{22}-a_{12} a_{21} \\
R_{2}=a_{11} a_{21}+a_{12} a_{22}
\end{array}\right.
$$

where $a_{11}=\sum_{n=b+1}^{N-b}[x(n) h(n)+\hat{x}(n) \hat{h}(n)]$,

$a_{12}=\sum_{n=b+1}^{N-b}[\hat{x}(n) h(n)-x(n) \hat{h}(n)]$,

$a_{21}=\sum_{n=b+1}^{N-b}[y(n) h(n)+\hat{y}(n) \hat{h}(n)]$,

$a_{22}=\sum_{n=b+1}^{N-b}[\hat{y}(n) h(n)-y(n) \hat{h}(n)]$.

When $\hat{\omega}=\omega$, the expectation of $R_{1}$ and $R_{2}$ are

$$
\begin{aligned}
& E\left[R_{1}\right]=(N-2 b)^{2} A B \sin \left(\theta_{2}-\theta_{1}\right) \\
& E\left[R_{2}\right]=(N-2 b)^{2} A B \cos \left(\theta_{2}-\theta_{1}\right)
\end{aligned}
$$

Therefore, estimated phase difference of two CMF signals are calculated as follows:

$$
\Delta \theta=\theta_{2}-\theta_{1}=\arctan \left(R_{1} / R_{2}\right)
$$

According to the analysis above, the basic procedure of the proposed method is as follows:

1) The coarse estimation frequency $\omega_{1}$ is calculated by utilizing (16), and frequency estimation $\hat{\omega}$ is gained by utilizing (6) and (11).

$$
\omega_{1}=\cos ^{-1}\left(\frac{b_{1}+\sqrt{b_{1}^{2}+8 c^{2}}}{4 c}\right)
$$

where $b_{1}=\sum_{k=1}^{p-4}[r(k)+r(k+4)] r(k+2)$,

$c=\sum_{k=1}^{p-4}[r(k+1)+r(k+3)] r(k+2)$. 
2) The quadrature-phase components of two CMF signals, $\hat{x}(n)$ and $\hat{y}(n)$, are calculated by (5).

3) Two reference signals, $h(n)$ and $\hat{h}(n)$, are generated in (13).

4) Two cross-correlation signals, $R_{1}$ and $R_{2}$, are calculated by (14).

5) Estimated phase difference $\theta_{2}-\theta_{1}$ is obtained by (15).

\section{Simulations}

To verify the effectiveness and superiority of the proposed method, simulations for the CC, HT, DEC, QDE methods and the proposed method are performed. In simulations, signal frequency is $146 \mathrm{~Hz}$, sampling frequency is $2000 \mathrm{~Hz}$,

$A=B=1, \theta_{1} \in[0,2 \pi), \theta_{2}=\theta_{1}+\frac{\pi}{6}$, the noise added into the signals is additive Gaussian white noise. All simulation results are the average of 1000 times of independent runs.

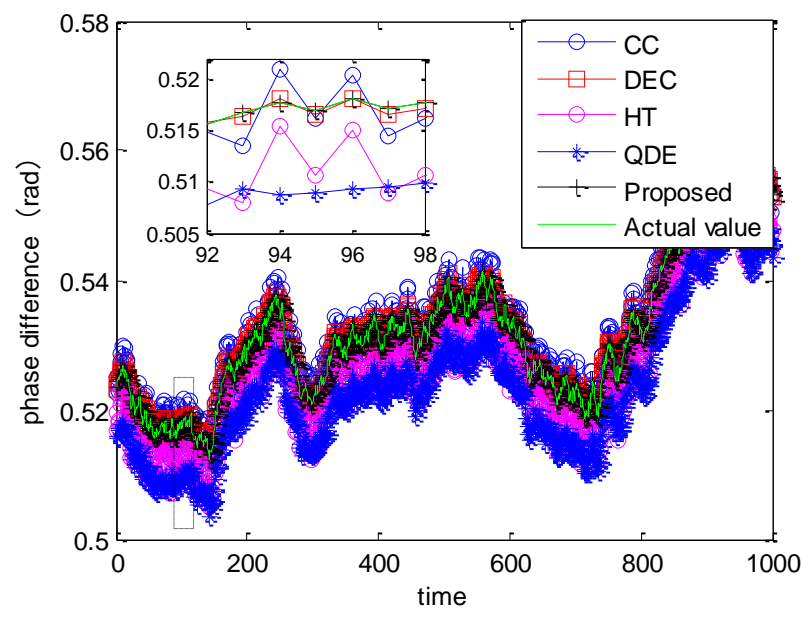

a) In the absence of noise

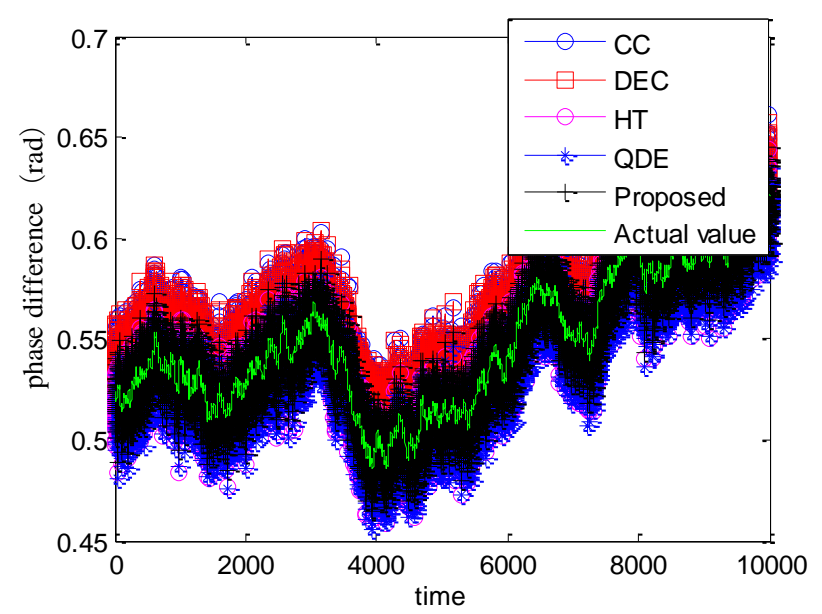

b) $\mathrm{SNR}=20 \mathrm{~dB}$
Fig.2. The results of phase difference estimation for different phase differences.

To evaluate the estimation performance of the proposed method under conditions of different phase differences, simulations at $N=200$ and $\Delta \theta \in(0.45,0.7) \mathrm{rad}$ are performed. The results of phase difference estimation are shown in Fig.2. It is shown in Fig.2.a) that the estimated phase differences of the proposed method equal actual values in the absence of noise while the CC, DEC, HT and QDE methods bring estimation errors. It demonstrates that the proposed method is unbiased in the absence of noise and the CC, DEC, HT and QDE methods are biased even though there is no noise. Fig.2.b) shows that the estimated values of the proposed method vary around the vicinity of actual phase differences, its errors remain in a small range. However, the estimated values of the other four methods are far from actual values and their phase difference estimation errors are obvious.

To evaluate the estimation performance of the proposed method under conditions of different SNRs, simulations at $N=200$ are performed. The mean square errors (MSE) of phase difference estimation are shown in Fig.3.

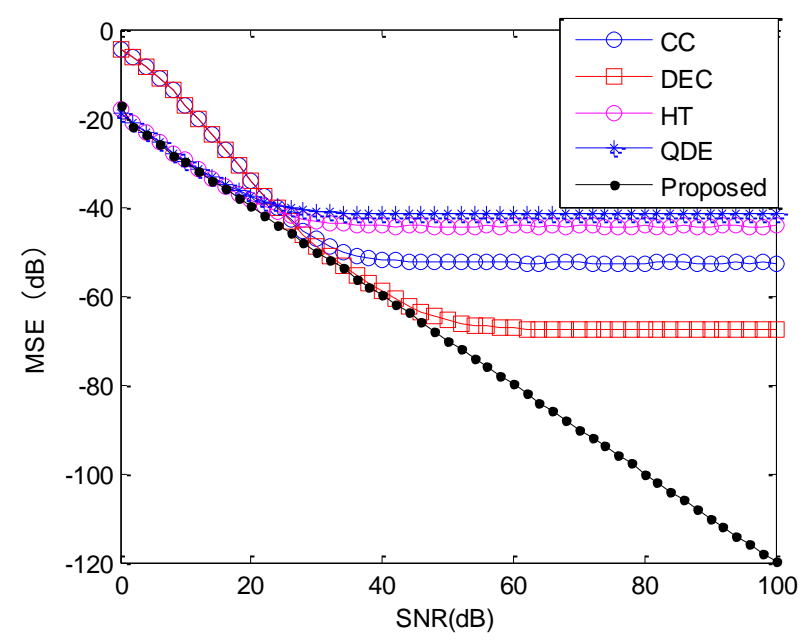

Fig.3. The results of phase difference estimation for different SNRs.

It is seen in Fig.3. that affected by non-integral period sampled signals, the phase difference estimation performance of the CC and HT methods is not improved when SNR is larger than $46 \mathrm{~dB}$ and $40 \mathrm{~dB}$, respectively. Compared with the CC method, the DEC method is less affected by non-integral period sampled signals. Due to the fact that phase shift of sinusoidal signals could not be exactly $\pi / 2$, the phase difference estimation of the QDE method is biased and its performance is not improved when SNR is larger than $36 \mathrm{~dB}$. The MSE of phase difference estimation for the proposed method are always smaller than those of the CC, HT, DEC and QDE methods under 
conditions of different SNRs. Besides, they are becoming smaller with the increasing of SNR.

To evaluate the estimation performance of the proposed method under conditions of different signal lengths, simulations at $\mathrm{SNR}=25 \mathrm{~dB}$ are performed. The MSE of phase difference estimation are shown in Fig.4. It is observed in Fig.4. that with the increasing of signal length, the MSE of the CC and HT methods show periodical damping oscillation. When $N \omega /(2 \pi) \approx m$ ( $m$ is an integer), that is to say, signal length satisfies integral period sampling, the MSE of the HT method are smaller than those of other signal lengths $N$. Likewise, when $N \omega / \pi \approx m_{1}\left(m_{1}\right.$ is an integer $)$, the MSE of the CC method are smaller. With the increase of signal length, the MSE of the proposed method decrease correspondingly. It demonstrates that the proposed method is not affected by non-integral period sampled signals. Furthermore, the MSE of the proposed method are always smaller than those of the QDE, CC, HT and DEC methods. Under conditions of different signal lengths, the average MSE of the proposed method are $4.8 \mathrm{~dB}, 3.2 \mathrm{~dB}, 2.8 \mathrm{~dB}$ and $2.1 \mathrm{~dB}$ smaller than those of the QDE, CC, DEC and HT methods, respectively.

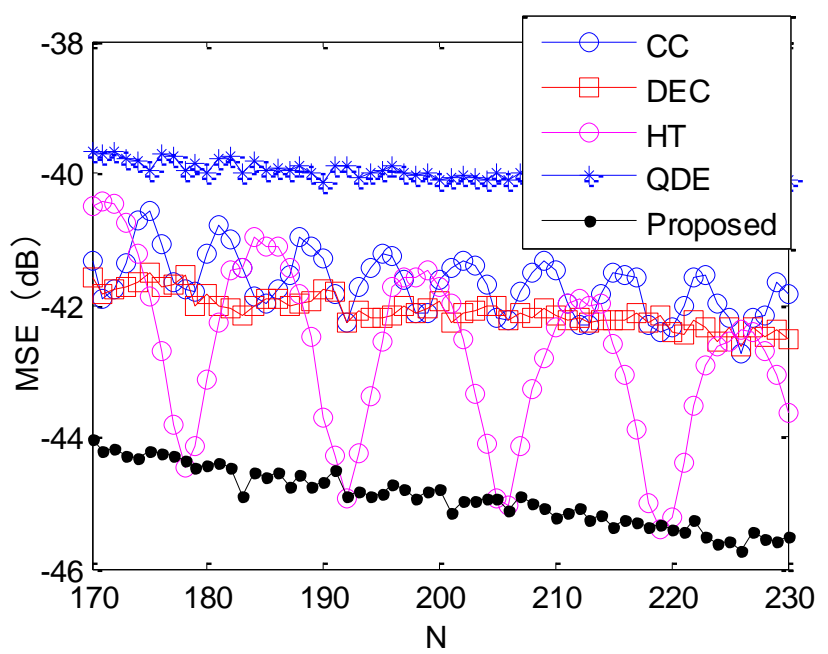

Fig.4. The results of phase difference estimation for different signal lengths.

\section{FIELD EXPERIMENTS}

In order to demonstrate the effectiveness of the proposed method to improve the measurement accuracy of CMF, a RHEONIK CMF with a RHE08 transmitter was used to test the estimation performance of the proposed method. In the experiments, the mass flow rates vary from 5 to $15 \mathrm{~kg} / \mathrm{min}$, the frequency of CMF sensors is about $146 \mathrm{~Hz}$ and the sampling frequency is $10 \mathrm{kHz}$. 4000 points of sampled signals are used to test the performance of the proposed method each time. The time interval $\Delta t$ is calculated as follows:

$$
\Delta t=\Delta \theta /\left(\hat{\omega} \cdot f_{s}\right)
$$

According to the principle of $\mathrm{CMF}$, there is a linear relationship between time interval $\Delta t$ and mass flow rate $M_{f}$.

$$
M_{f}=B_{0} \cdot \Delta t+C_{0}
$$

where the coefficient $B_{0}$ is a fixed constant on the same experimental condition, which is dependent on the CMF type, measured medium, pipe material, etc. Before the experiments, the CMF with a RHE08 transmitter is calibrated in a qualified calibration laboratory. Therefore, the mass flow rates shown by the $\mathrm{CMF}$ are deemed as true values. The experimental results are shown in Table 1. The results demonstrate that for different flow rates, the estimated mass flow rates of the proposed method are closer to the actual values than those of the CC, HT, DEC, QDQ and QDE methods. The average relative error of the estimated mass flow rates for the proposed method is about $0.3 \%$, which is smaller than those of the CC, HT, DEC, QDQ and QDE methods. Therefore, the proposed method contributes to improving the estimation precision of phase difference and the measurement accuracy of the CMF.

Table 1. Actual and estimated mass flow rates.

\begin{tabular}{|c|c|c|c|c|c|c|c|}
\hline & \multirow{2}{*}{$\begin{array}{c}\text { Mass flow rates } \\
(\mathrm{kg} / \mathrm{min})\end{array}$} & \multicolumn{6}{|c|}{ Estimated mass flow rates(kg/min ) } \\
\cline { 3 - 8 } & & DEC & HT & DQD & QDE & Proposed \\
\hline 1 & 5.88 & 8.246 & 8.269 & 5.867 & 5.900 & 5.867 & 5.870 \\
\hline 2 & 8.50 & 10.186 & 10.189 & 8.437 & 8.460 & 8.452 & 8.481 \\
\hline 3 & 10.34 & 11.691 & 11.699 & 10.128 & 10.154 & 10.280 & 10.310 \\
\hline 4 & 12.13 & 13.491 & 13.453 & 12.029 & 12.089 & 12.080 & 12.105 \\
\hline 5 & 13.95 & 15.075 & 15.073 & 13.885 & 13.909 & 13.893 & 13.920 \\
\hline
\end{tabular}




\section{CONCLUSIONS}

To improve the signal processing precision of $\mathrm{CMF}$ signals and the measurement accuracy of $\mathrm{CMF}$, a phase and frequency matching-based signal processing method for Coriolis mass flowmeters is proposed. The proposed method obtains estimated phase difference and mass flow rates by means of frequency estimation, $90^{\circ}$ phase shift, generating reference signals and cross-correlation. Theoretical analysis demonstrates that the proposed method has completed $90^{\circ}$ phase shift exactly and obtained unbiased phase difference estimation without the special requirement of integral period sampling or specific sampling frequency. Simulated results under conditions of different phase differences, SNRs and signal lengths show that the proposed method is not affected by non-integral period sampled signals. Besides, the proposed method has better estimation performance of phase difference than the CC, HT, DEC and QDE methods. The experimental results of mass flow rates for CMF verify the effectiveness and superiority of the proposed method.

\section{ACKNOWLEDGMENTS}

The study is supported by National Natural Science Foundation of China (NNSFC) (Grant Nos. 61271449, 61302175), Natural Science Foundation of Chongqing (Grant No. CSTC2015jcyjBX0017) and Postgraduate research innovation project of Chongqing (Grant No. CYB14100).

\section{REFERENCES}

[1] Hou, Q., Xu, K., Fang, M., Liu, C., Xiong, W. (2013). Development of Coriolis mass flowmeter with digital drive and signal processing technology. ISA Transactions, 52 (5), 692-700.

[2] Svete, A., Kutin, J., Bobovnik, G., Bajsić, I. (2015). Theoretical and experimental investigations of flow pulsation effects in Coriolis mass flowmeters. Journal of Sound and Vibration, 352, 30-45.

[3] Shanmugavalli, M., Umapathy, M., Uma, G. (2010). Smart Coriolis mass flowmeter. Measurement, 43 (4), 549-555.

[4] Romano, P. (1990). Coriolis mass flow rate meter having a substantially increased noise immunity. US Patent 4934196.

[5] Assous, S., Linnett, L. (2012). High resolution time delay estimation using sliding discrete Fourier transform. Digital Signal Processing, 22 (5), 820-827.
[6] Shaswary, E., Tavakkoli, J., Xu, Y. (2015). A new algorithm for time-delay estimation in ultrasonic echo signals. IEEE Transactions on Ultrasonics Ferroelectrics and Frequency Control, 62 (1), 236241.

[7] Ren, H., Yin, W., Hu, F. (2010). Contrast and analysis of three measuring methods for phase difference based on labview. Science Technology and Engineering, 10 (1), 263-268.

[8] Li, J., Zhao, P., Hou, W., Zheng, B., Zheng, H. (2009). Research on error of phase difference algorithm based on correlation theory. Journal of North University of China, 30 (6), 616-619.

[9] Shen, T., Tu, Y., Li, M., Zhang, H. (2014). Research and validation on improved correlation method for phase difference measurement based on data extension. Chinese Journal of Scientific Instrument, 35 (6), 1331-1337.

[10] Yang, H., Tu, Y., Zhang, H., Yi, P. (2012). Phase difference measuring method based on SVD and Hilbert transform for Coriolis mass flowmeter. Chinese Journal of Scientific Instrument, 33 (9), 21012107.

[11] Liu, W., Zhao, L., Wang, K., Feng, Z., Long, Q. (2013). Signal processing for Coriolis mass flowmeter based on Hilbert transform. Acta Metrologica Sinica, 34 (5), 446-451.

[12] Tu, Y., Yang, H., Zhang, H., Liu, X. (2014). CMF signal processing method based on feedback corrected ANF and Hilbert transformation. Measurement Science Review, 14 (1), 41-47.

[13] Vučijak, N.M., Saranovac, L.V. (2010). A simple algorithm for the estimation of phase difference between two sinusoidal voltages. IEEE Transactions on Instrumentation and Measurement, 59 (12), 31523158.

[14] Maskell, D.L., Woods, G.S. (2005). Adaptive subsample delay estimation using a modified quadrature phase detector. IEEE Transactions on Circuits and Systems II: Express Briefs, 52 (10), 669674.

[15] So, H.C. (2005). A comparative study of two discretetime phase delay estimators. IEEE Transactions on Instrumentation and Measurement, 54 (6), 2501-2504.

Received November 2, 2015. Accepted March 23, 2016. 